Supporting information for

\title{
Noncovalently Caged Firefly Luciferins Enable Amplifiable Bioluminescence Sensing of Hyaluronidase-1 Activity in Vivo
}

Jinqiu Luo, ${ }^{\dagger, \#}$ Jinfeng Yang, ${ }^{\ddagger, \#}$ Guangjie Li, ${ }^{\dagger, \#}$ Sheng Yang, ${ }^{\dagger, *}$ Yibo Zhou, ${ }^{\dagger}$ Jun-Bin Li, ${ }^{\dagger}$

Ge Huang, ${ }^{\ddagger}$ Yibo Hu, ${ }^{\S}$ Shuangfa Zou, ${ }^{\ddagger}$ Qinghai Zeng,,${ }^{\S}$ and Ronghua Yang ${ }^{\dagger, *}$

†Hunan Provincial Key Laboratory of Cytochemistry, School of Chemistry and Food Engineering, Changsha University of Science and Technology, Changsha, 410114, P.

R. China

Hunan Cancer Hospital/the Affiliated Cancer Hospital of Xiangya School of Medicine, Central South University, Changsha, 410083, P. R. China

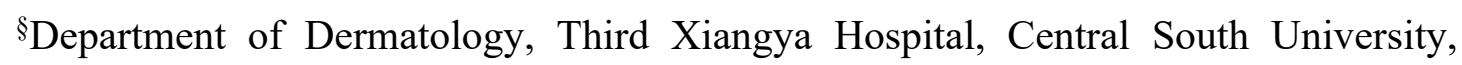
Changsha, 410013, P. R. China

\#These authors contributed equally to this work.

*To whom correspondence should be addressed:

E-mail: yangrh@pku.edu.cn

Fax: +86-731-8882 2523 


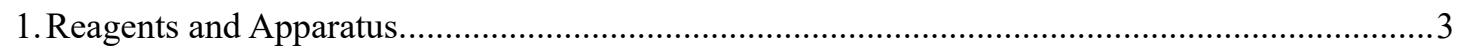

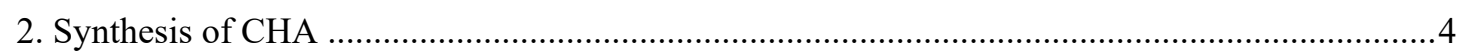

3. Calculations on the Loading Capacity and Cumulative Release ..................................................

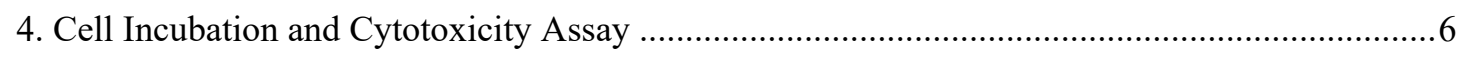

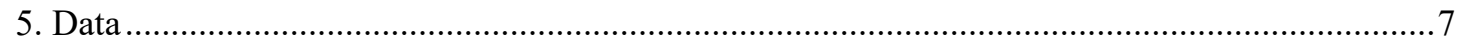

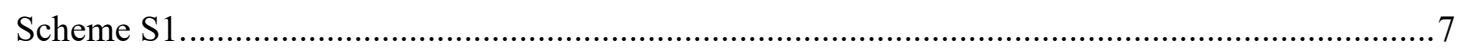

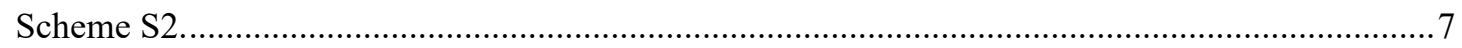

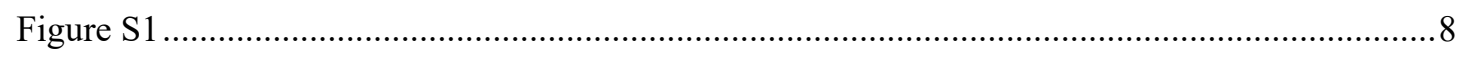

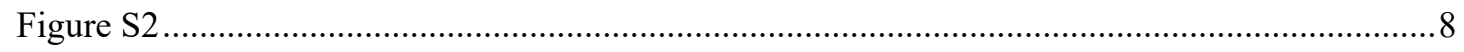

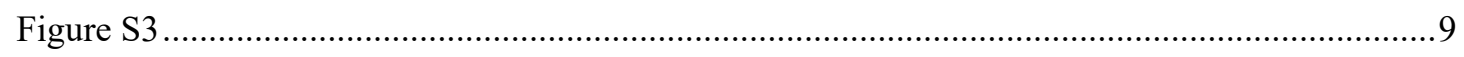

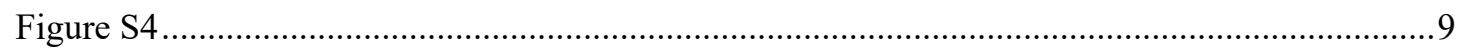

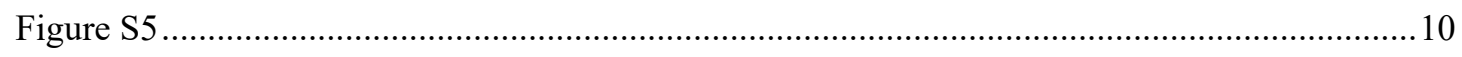

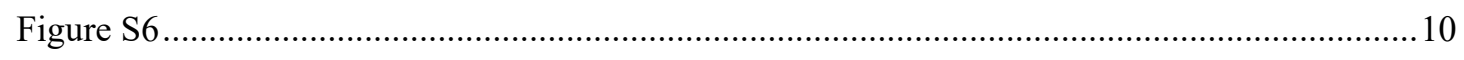

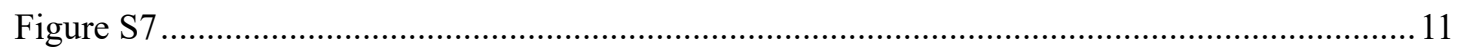

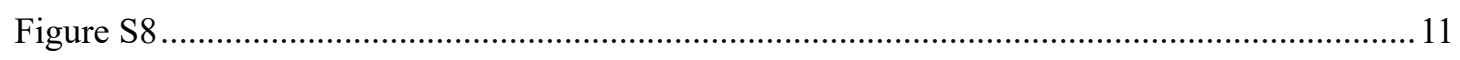

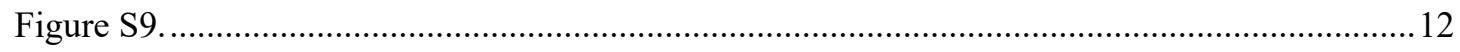

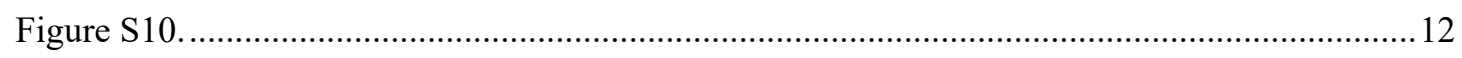

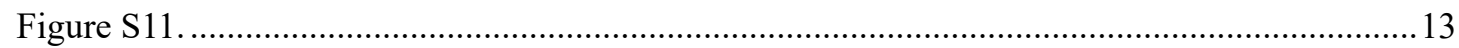

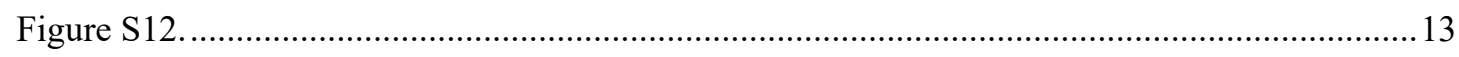

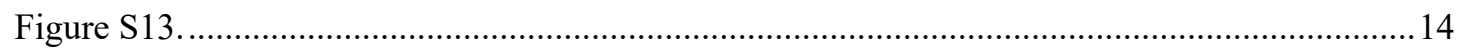

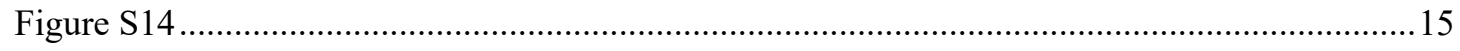

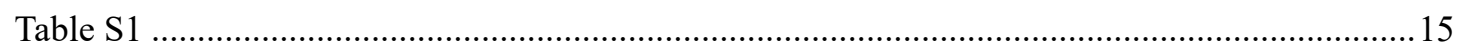




\section{Reagents and Apparatus.}

All chemicals were purchased from commercial suppliers (J\&K Scientific, TCI, and Aladdin). Firefly luciferase was purchased from Promega. Hyal-1 was purchased from R\&D Systems and other Hyals (Hyal-2, Hyal-3, Hyal-4 and PH-20) were purchased from Proteintech. Ultrapure water used in all experiments was purified via a Milli-Q water system (Millipore, U.S.A.). Human breast cancer cell line transfected with fLuc (MDA-MB-231-fLuc) and fLuc-transfected cells line 4T1 (4T1-fLuc) were generously provided by Professor X. B. Zhang at Hunan University (Changsha, China). Mice were obtained from Hunan SJA Laboratory Animal Co., Ltd (Changsha, China).

UV-vis absorption spectra were measured by using a UV-2700 spectrophotometer (Shimadzu, Japan). NMR spectra were recorded on a Bruker DRX-400 spectrometer (Bruker, Germany). Mass spectrometry analysis was performed by an LCQ-Advantage and MAT 95 XP spectrometer (Thermo Finnigan, U.S.A.). Dynamic light scattering (DLS) measurements and Zeta-potential were accomplished on a Nano-ZS 90/ZS (Malvern, Britain). Transmission electron microscopy (TEM) images were obtained from a Tecnai G2 20 (FEI, U.S.A.). Luminescence spectra were conducted on a F-7000 fluorescence system (Hitachi, Japan) with the Xe lamp shut off. The $\mathrm{pH}$ was measured with a PHS-3C $\mathrm{pH}$ meter (Shanghai INESA Scientific Instrument Co., Ltd., China). MTT assay was carried on a Synergy 2 Multi-Mode Microplate Reader (Bio-Tek Instruments, Inc, U.S.A.). The

IVIS Lumina III System (PerkinElmer, U.S.A.) equipped with a cooled 
charge-coupled device (CCD) camera was manipulated for bioluminescence images in vitro, cells, and vivo. UV-B irradiation was used an Ultraviolet Phototherapy Instrument (SS-03) (Shanghai Sigma High-Tech Co., Ltd., China).

\section{Synthesis of CHA}

First, Cholesterol chloroformate (L-Chol, $400 \mathrm{mg}$ ) was dissolved in dichloromethane DCM $(20 \quad \mathrm{~mL})$ and added dropwise to 2,2'-(ethylenedioxy)-bis-ethylamine (1.5 g) containing triethylamine (500 mg). The solution of anhydrous DCM (30 mL) was added dropwise with ice-cooling for 30 minutes under nitrogen, then returned to room temperature and stirred overnight. Then, the product amino-L-Chol was isolated following column chromatography on silica gel using a stepwise methanol gradient (5\%) in DCM. Molecular weight of amino-L-Chol, which was dissolved in DCM, was performed by using Electrospray Ionization Mass Spectrometry (ESI-MS). Second, synthesis of CHA by activation of HA (500 mg), dissolved in warm anhydrous dimethyl sulfoxide (DMSO) (15 mL) using 1-ethyl-3-(3-dimethylaminopropyl) carbodiimide (EDC) (15 mg), hydroxybenzotriazole (HOBT) $(10 \mathrm{mg})$, TEA $(20 \mu \mathrm{L})$ and 4-dimethylaminopyridine (DMAP, $2.5 \mathrm{mg}$ ) at continuous stirring under nitrogen. After activation at $25^{\circ} \mathrm{C}$ for 4 hours, amino-L-choline $(70 \mathrm{mg})$ dissolved in DMSO $(2.5 \mathrm{~mL})$ was added, and the reaction mixture was stirred at $25^{\circ} \mathrm{C}$ for 48 hours. The final product (CHA) was dialyzed against water three times in a semi-permeable tube (MWCO 12-14 kDa) at $25^{\circ} \mathrm{C}$ for 24 hours and then lyophilized. 


\section{Calculations on the Loading Capacity and Cumulative Release}

The amount of D-Luciferin loaded in CHA was determined by UV-vis spectrophotometry. The absorbance at $320 \mathrm{~nm}$ of nanosensor was firstly measured, and then the concentration of D-Luc loaded into CHA (4-fold dilution) were obtained from a calibration curve, which was linear over the concentration of D-Luc from 5 $\mu \mathrm{M}$ to $100 \mu \mathrm{M}$ with a correlation coefficient of $\mathrm{R}^{2}=0.997$ (as shown in Figure $\mathrm{S} 4 \mathrm{~B}$ ). Finally, loading capacity (LC) can be calculated by the following equation:

$$
\mathrm{LC}=\mathrm{M}_{\mathrm{e}} /\left(\mathrm{W}_{\mathrm{m}}+\mathrm{W}_{\mathrm{e}}\right)
$$

Where $M_{e}$ represents amount of D-Luc in nanosensor; $W_{m}$ and $\mathbf{W}_{\mathbf{e}}$ are weight of CHA and D-Luc in nanosensor, respectively.

The cumulative release was calculated by modified literature methods, a sample of 0.2 mg D-Luc@CHA was placed into a beaker containing 0.5 mL PBS (10 mM, pH 7.4) and same concentrations of Hyals at $37^{\circ} \mathrm{C}$ under magnetic stirring $500 \mu \mathrm{L}$ of solution was collected from the beaker at various times and centrifuged at 10,000 rpm for 3 min at $4{ }^{\circ} \mathrm{C}$, followed by measurement of the supernatant using UV-vis spectrophotometer. To maintain the total volume, the same volume of buffer was replenished after each collection. All experiments were carried out in triplicate. The accumulative release (AR) was calculated by the following equation:

$$
\operatorname{Er}(\%)=\frac{V e \sum_{I}^{n-1} C_{i}+V_{0} C_{n}}{m_{e}} \times 100
$$

Where $\mathrm{V}_{\mathrm{e}}$ and $\mathrm{V}_{0}$ represent volume of replacement PBS and whole volume of the release medium, respectively; $C_{i}$ and $C_{n}$ are concentrations of D-Luc in the releasing 
medium when times $\mathrm{i}$ and $\mathrm{n}$ replaced PBS; $\mathrm{M}_{\mathrm{e}}$ represents the amount of D-Luc in CHA.

\section{Cell Incubation and Cytotoxicity Assay}

First, fLuc-transfected MDA-MB-231 cells in the logarithmic growth phase were taken, trypsinized, centrifuged (centrifugation rate: $2000 \mathrm{rpm}, 8 \mathrm{~min}$ ), and the lower cells were incubated in a single cell dispersion state with fresh culture liquid. Liquid (number of cells: $5 \times 10^{4} / \mathrm{mL}$ ). Next, MDA-MB-231-fLuc cells were separately added to each well of a 96-well culture plate (the edge wells were filled with the same volume of sterile $1 \times \mathrm{PBS}$ buffer), and cultured in a $37^{\circ} \mathrm{C} 5 \% \mathrm{CO}_{2}$ incubator for $24 \mathrm{~h}$ to allow the cells to grow. Adhere well, discard the old medium, wash once with sterile PBS buffer, and add nanosensors with different concentrations $(0,0.05,0.5,1.0$, 2.0, 2.0, 4.0, $6.0 \mathrm{mg} / \mathrm{mL}$ ). The fresh medium was incubated for $24 \mathrm{~h}$. Then $10 \mu \mathrm{L}$ of MTT reagent was added to the above solution, and incubation was continued for $4 \mathrm{~h}$ in an incubator. After carefully discarding the supernatant, add $150 \mu \mathrm{L}$ of dimethyl sulfoxide (DMSO) to each well, and immediately measure the absorbance of each well using a microplate reader. Set 5 replicate wells for all concentrations and take five replicate wells. average value. 


\section{Data}
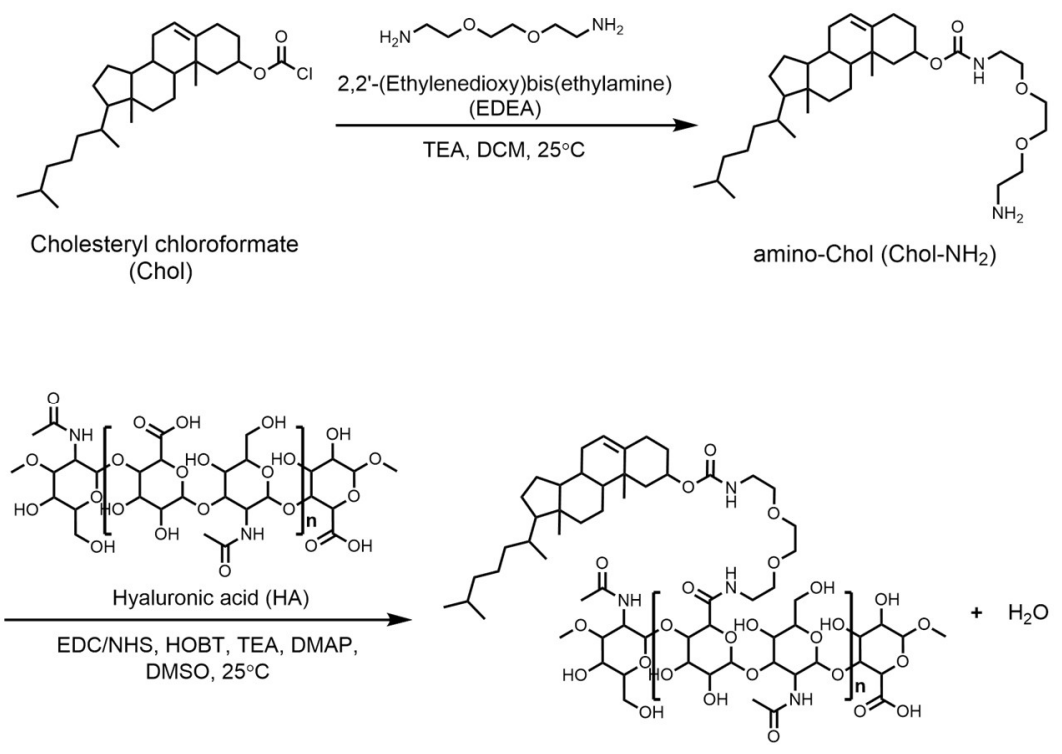

Chol-HA (CHA)

Scheme S1. Synthesis route of Chol-HA.

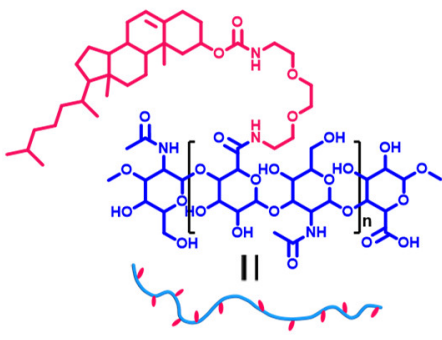

Chol-HA

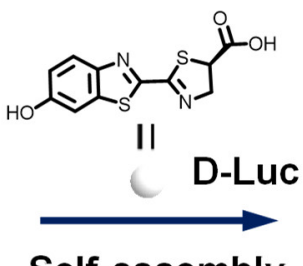

Self-assembly

Scheme S2. Synthesis of D-Luc@CHA by self-assembly of Chol-HA and D-Luc. 


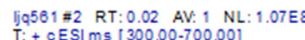

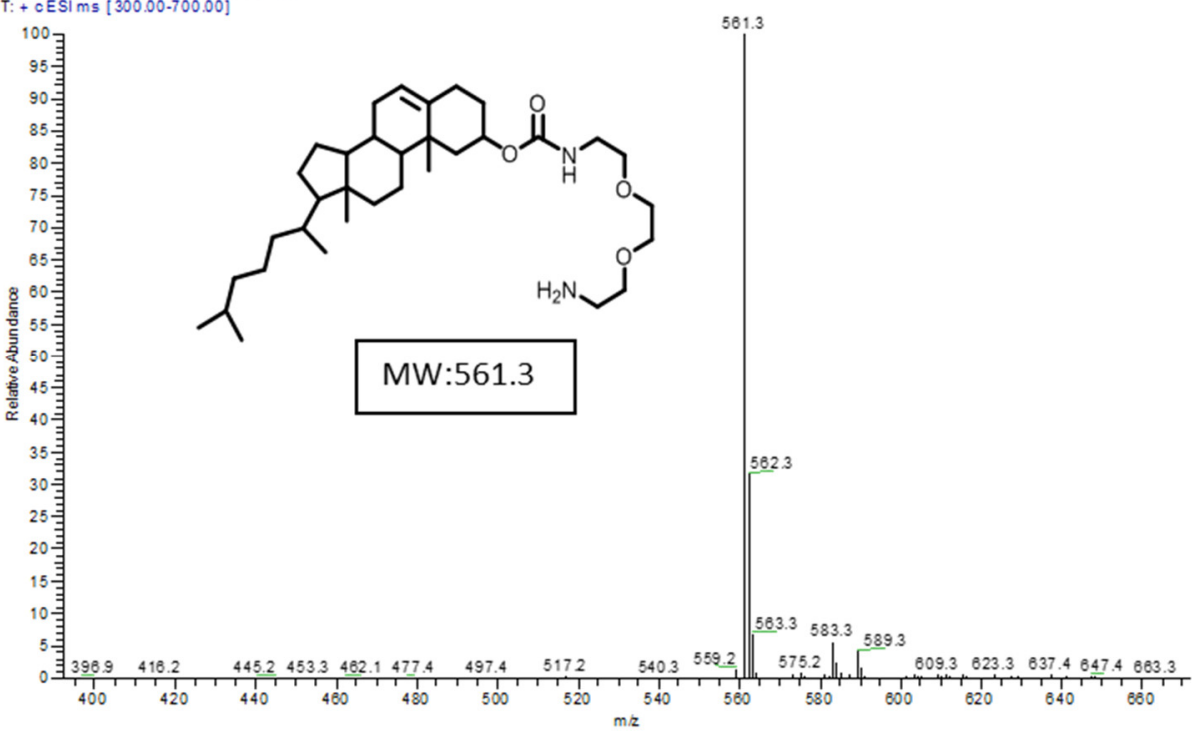

Figure S1. ESI-MS of Chol-NH2.

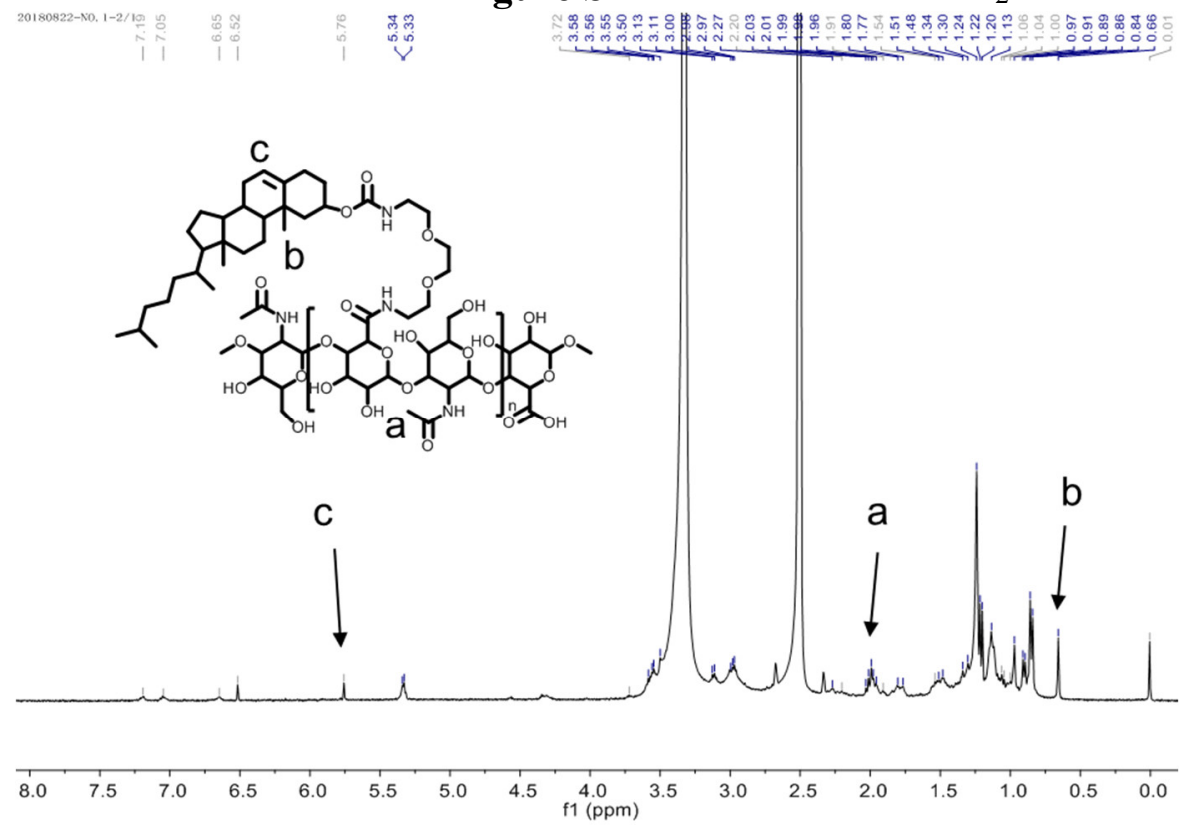

Figure S2. ${ }^{1} \mathrm{H}-\mathrm{NMR}(500 \mathrm{MHz})$ spectrum of Chol-HA in DMSO-d 6 . 


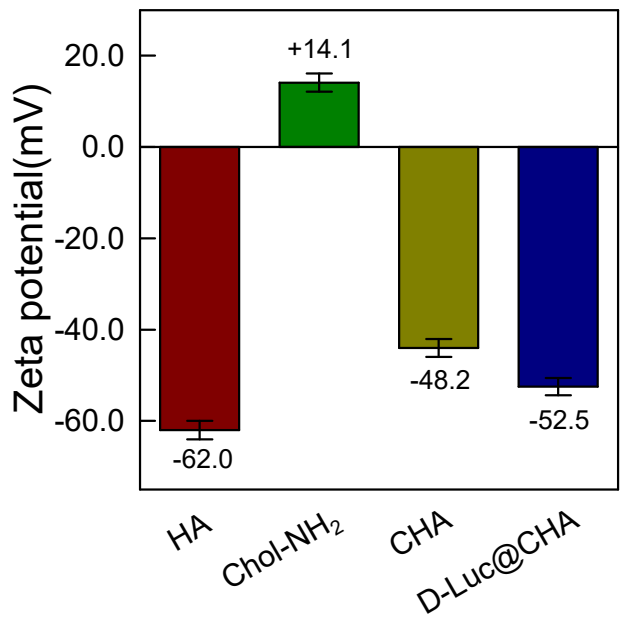

Figure S3. Zeta potenial of HA, Chol-NH 2, CHA, D-Luc@CHA. Error bars indicate s.d. $(n=3)$.
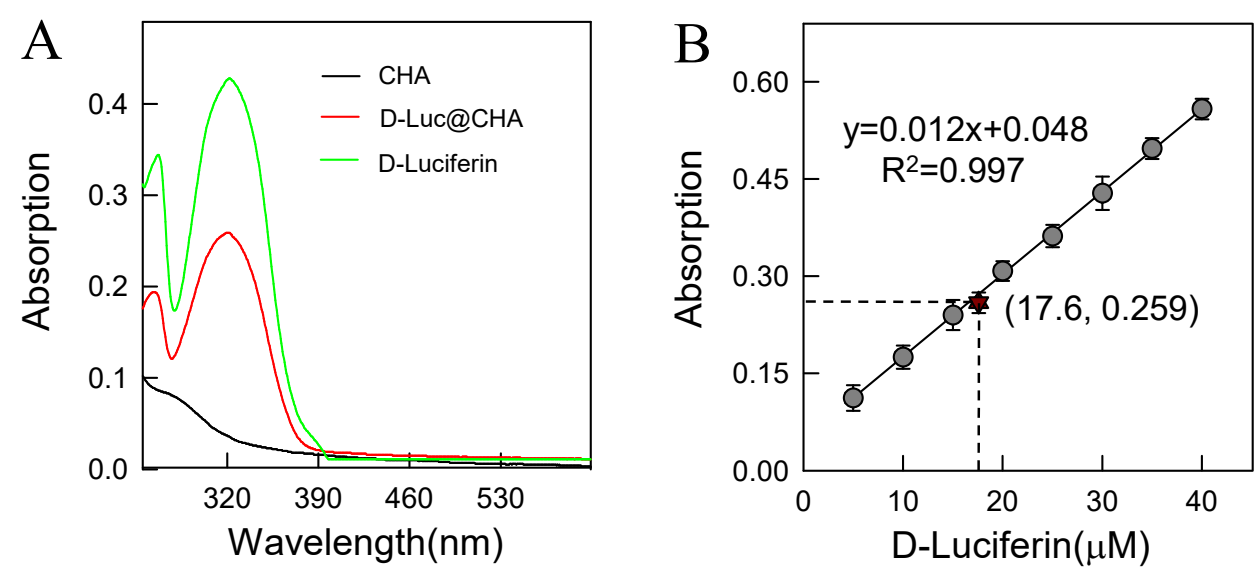

Figure S4. Absorption strength of added D-luciferin and loaded D-Luciferin. (A) Absorption spectra of CHA (0.1mg/mL), D-Luc@CHA (0.1mg/mL), D-Luciferin (30 $\mu \mathrm{mol} / \mathrm{mL}$ ). (B) The calibration curve of D-Luciferin from $5 \mu \mathrm{M}$ to $40 \mu \mathrm{M}$. Error bars represent $\pm \operatorname{SD}(n=3)$. 


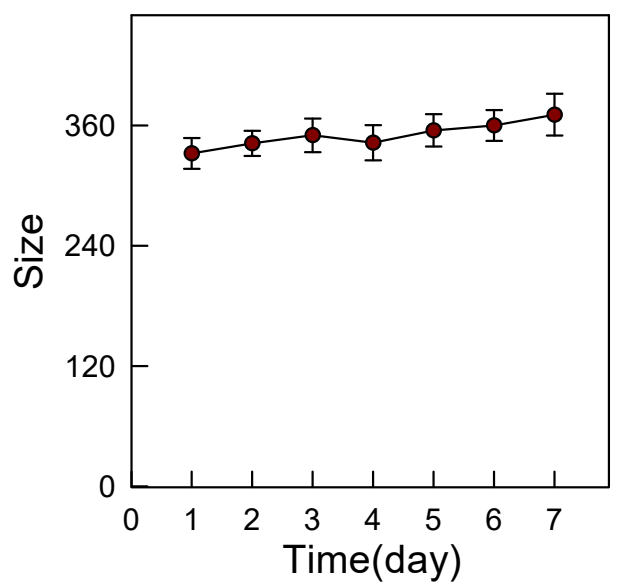

Figure S5. The size change of the nanosensor varies with the time in buffer solution.

Error bars indicate s.d. $(\mathrm{n}=3)$.

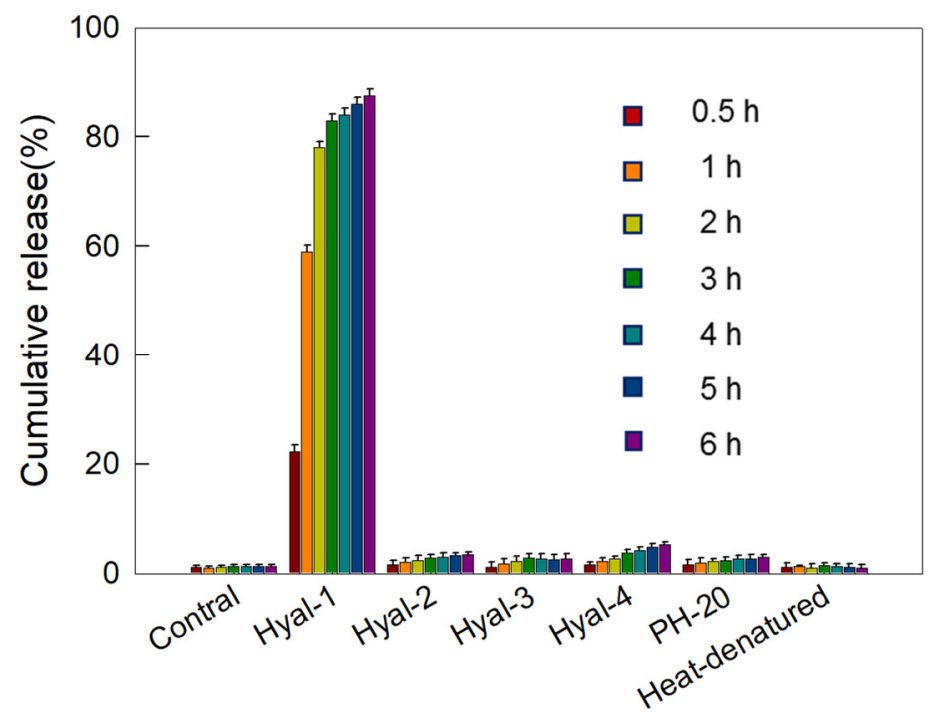

Figure S6. Cumulative release of nanosensor $(0.3 \mathrm{mg} / \mathrm{mL})$ before and after Hyal-1, Hyal-2, Hyal-3, Hyal-4, PH-20, heat-denatured Hyal-1 $(5 \mu \mathrm{g} / \mathrm{mL})$ in PBS (10 mM) treatments at $0.5,1,2,3,4,5,6 \mathrm{~h}$. 

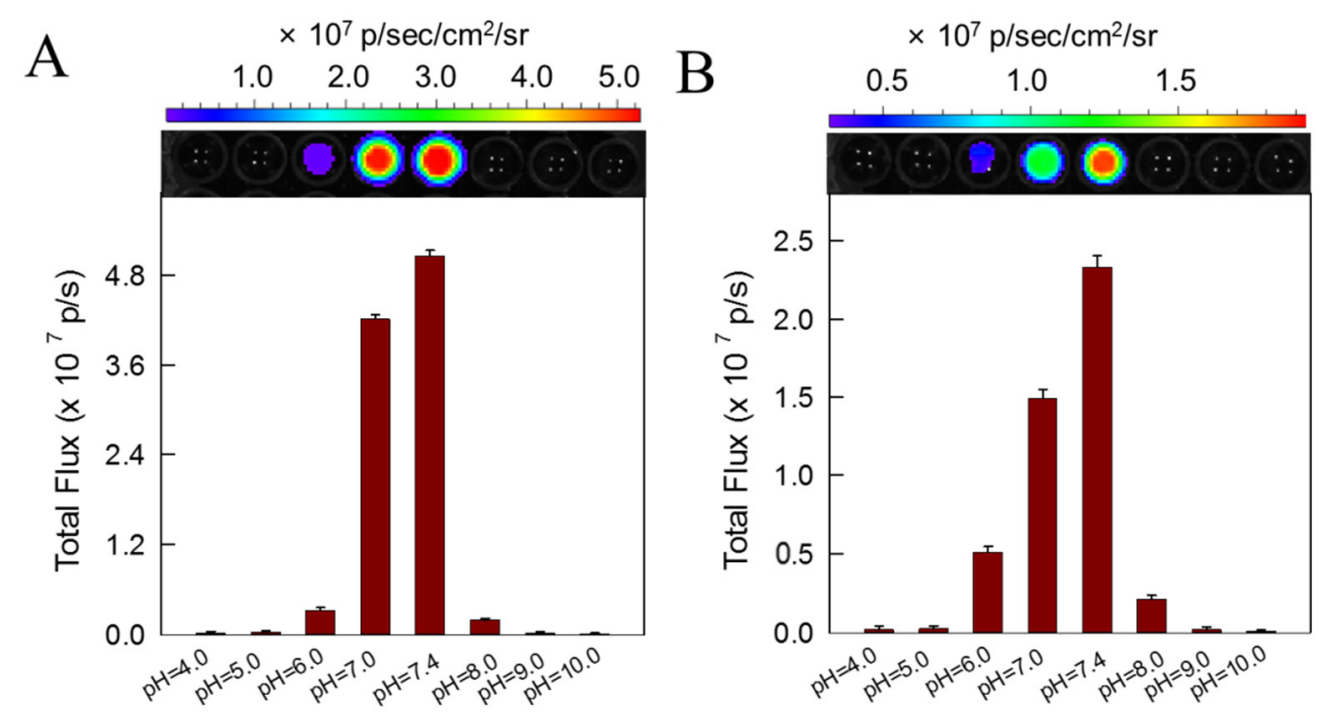

Figure S7. Bioluminescence image and the corresponding total flux (photon/s) of 20 $\mu \mathrm{M}$ D-Luciferin (A) and $0.3 \mathrm{mg} / \mathrm{mL}$ D-Luc@CHA with $5 \mu \mathrm{g} / \mathrm{mL}$ Hyal-1 (B) at different $\mathrm{pH}$ values $(\mathrm{pH}=4.0,5.0,6.0,7.0,7.4,8.0,9.0,10.0)$. Error bars represent \pm $\operatorname{SD}(n=3)$.

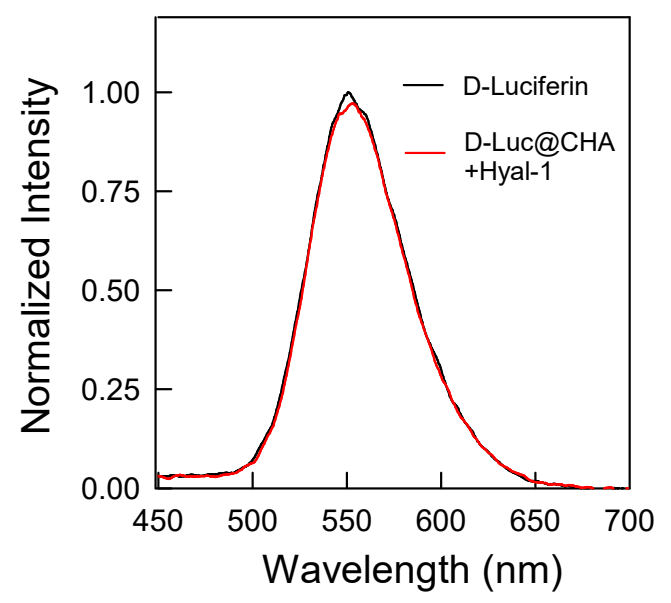

Figure S8. Normalized bioluminescence spectra of Luciferin and D-Luc@CHA with and without Hyal-1 $([$ Hyal-1] $=5 \mu \mathrm{g} / \mathrm{mL},[$ Luciferin $]=15 \mu \mathrm{M},[\mathrm{D}-\mathrm{Luc} @ \mathrm{CHA}]=0.3$ mg/mL). Signals were normalized to the signal from D-Luc@CHA treated with Hyal-1 


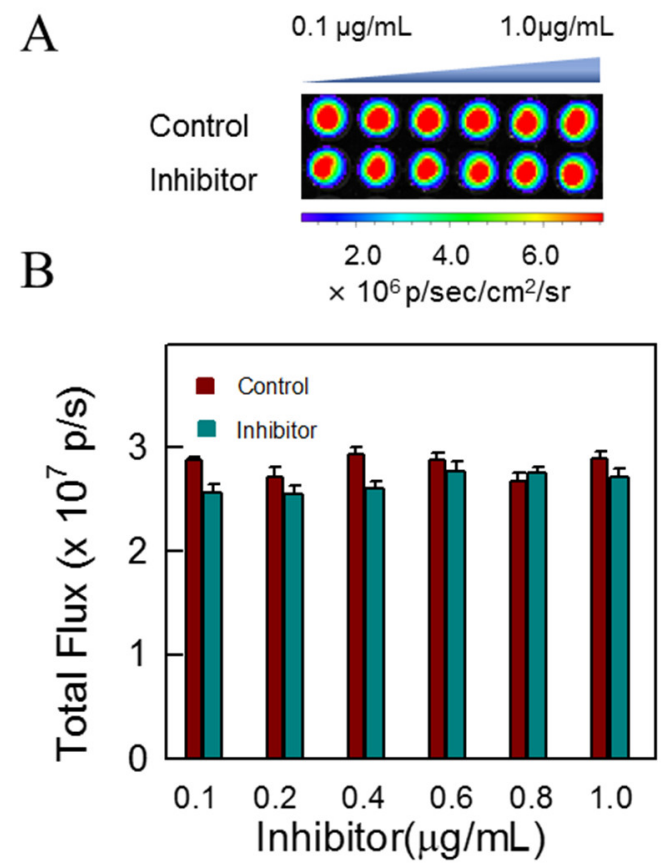

Figure S9. (A) bioluminescence imaging of $20 \mu \mathrm{M}$ D-Luciferin in the presence or absence of different concentrations indomethacin $(0.1,0.2,0.4,0.6,0.8,1.0 \mu \mathrm{g} / \mathrm{mL})$ in PBS (10 mM, pH 7.4) containing $10 \mu \mathrm{g} / \mathrm{mL}$ fLuc, $2 \mathrm{mM} \mathrm{ATP}$, and $10 \mathrm{mM} \mathrm{Mg}{ }^{2+}$. (B) Quantification of the total flux (photon/s) in the various component. Error bars represent $\pm \operatorname{SD}(\mathrm{n}=3)$.

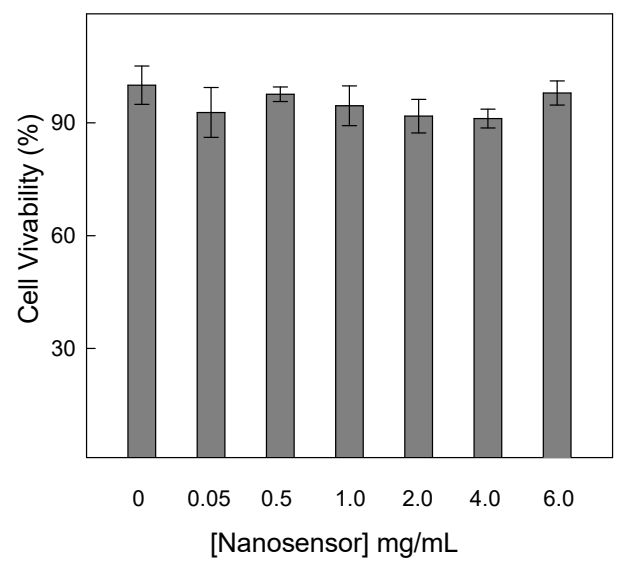

Figure S10. MTT assay of nanosensor D-Luc@CHA on MDA-MB-231-fLuc cells.

The cells were incubated for $24 \mathrm{~h}$. The results are the mean \pm standard deviation of five separate measurements. 
A

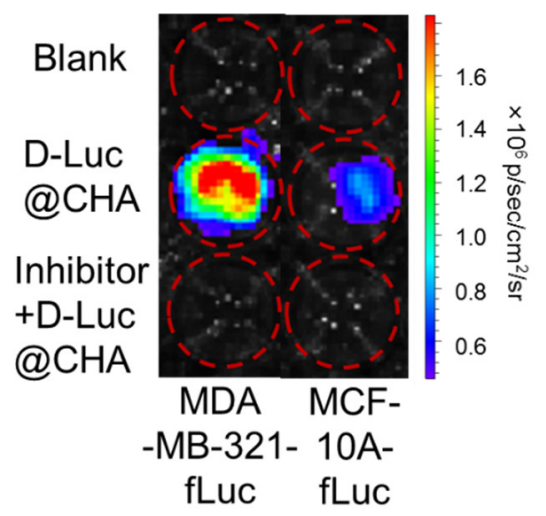

$\mathrm{B}$

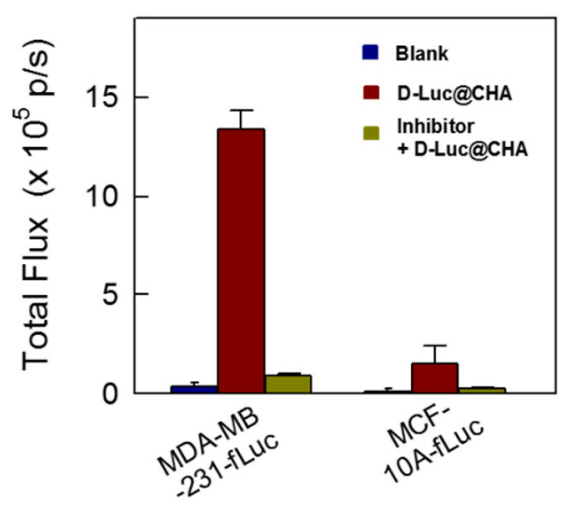

Figure S11. (A) Bioluminescence imaging of fLuc-transfected MDA-MB-231 and MCF-10A cells incubated with PBS buffer solution (10 mM, pH 7.4), preincubated with D-Luc@CHA, preincubated with $0.8 \mu \mathrm{g} / \mathrm{mL}$ indomethacin and then with 0.3 mg/mL D-Luc@CHA acquired at 90 min in serum-free culture medium at $37^{\circ} \mathrm{C}$. (B) Quantification of the total flux (photon/s) in the various component. Error bars represent $\pm \operatorname{SD}(n=3)$.

A

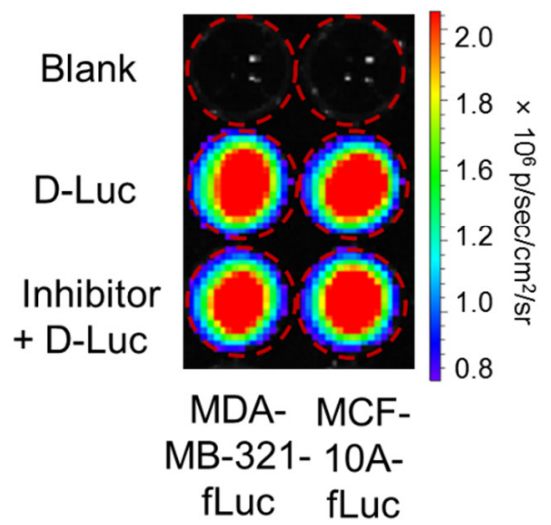

$\mathrm{B}$

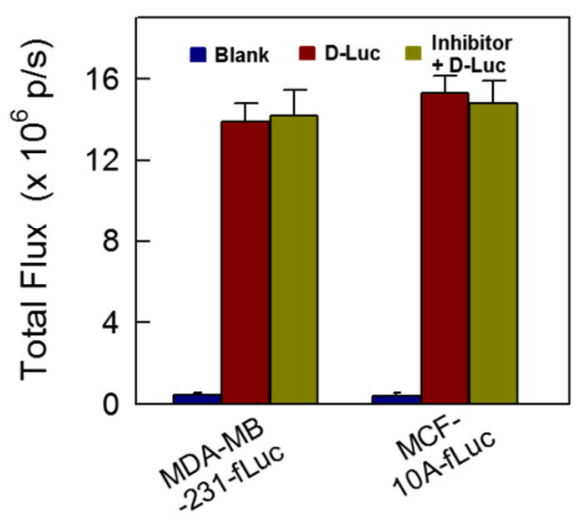

Figure S12. (A) Bioluminescence imaging of fLuc-transfected MDA-MB-231 and MCF-10A cells incubated with PBS buffer solution (10 mM, $\mathrm{pH} 7.4)$, incubated with $10 \mu \mathrm{M}$ D-Luciferin, preincubated with $0.8 \mu \mathrm{g} / \mathrm{mL}$ indomethacin and then with $10 \mu \mathrm{M}$ D-Luciferin acquired at $5 \mathrm{~min}$ in serum-free culture medium at $37{ }^{\circ} \mathrm{C}$. (B) 
Quantification of the total flux (photon/s) in the various component. Error bars represent $\pm \operatorname{SD}(n=3)$.

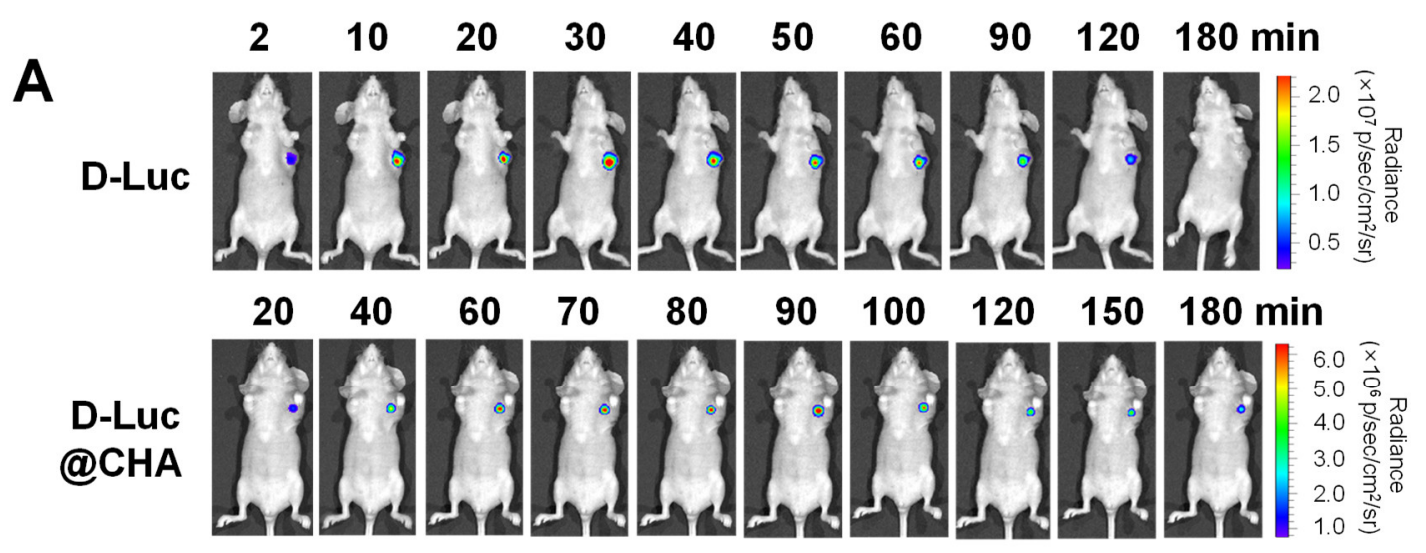

B

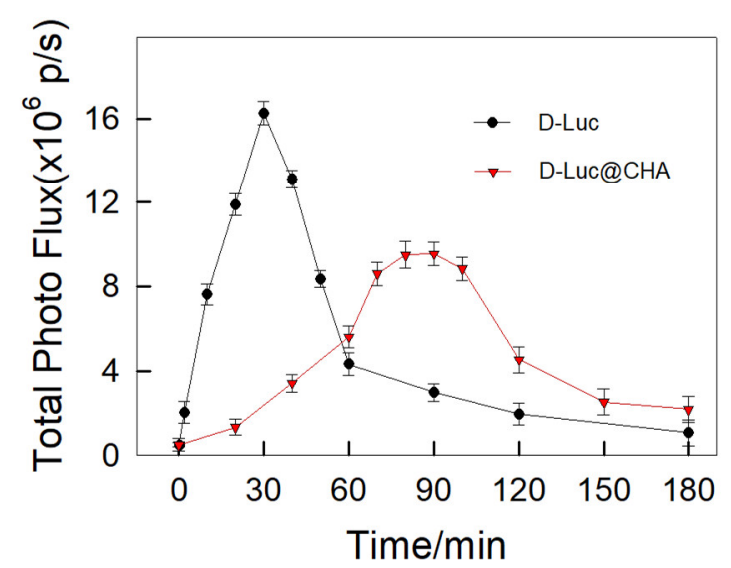

Figure S13. (A) Time-course bioluminescence imaging of nude mice after intratumor injection of D-Luc $(1 \mathrm{mM}, 100 \mu \mathrm{L})$ or D-Luc@CHA $(0.3 \mathrm{mg} / \mathrm{mL}, 100 \mu \mathrm{L})$. (B) Quantification of the total flux (photon/s) from the tumor regions for the mouse images (A). Error bars represent $\pm \operatorname{SD}(n=3)$. 


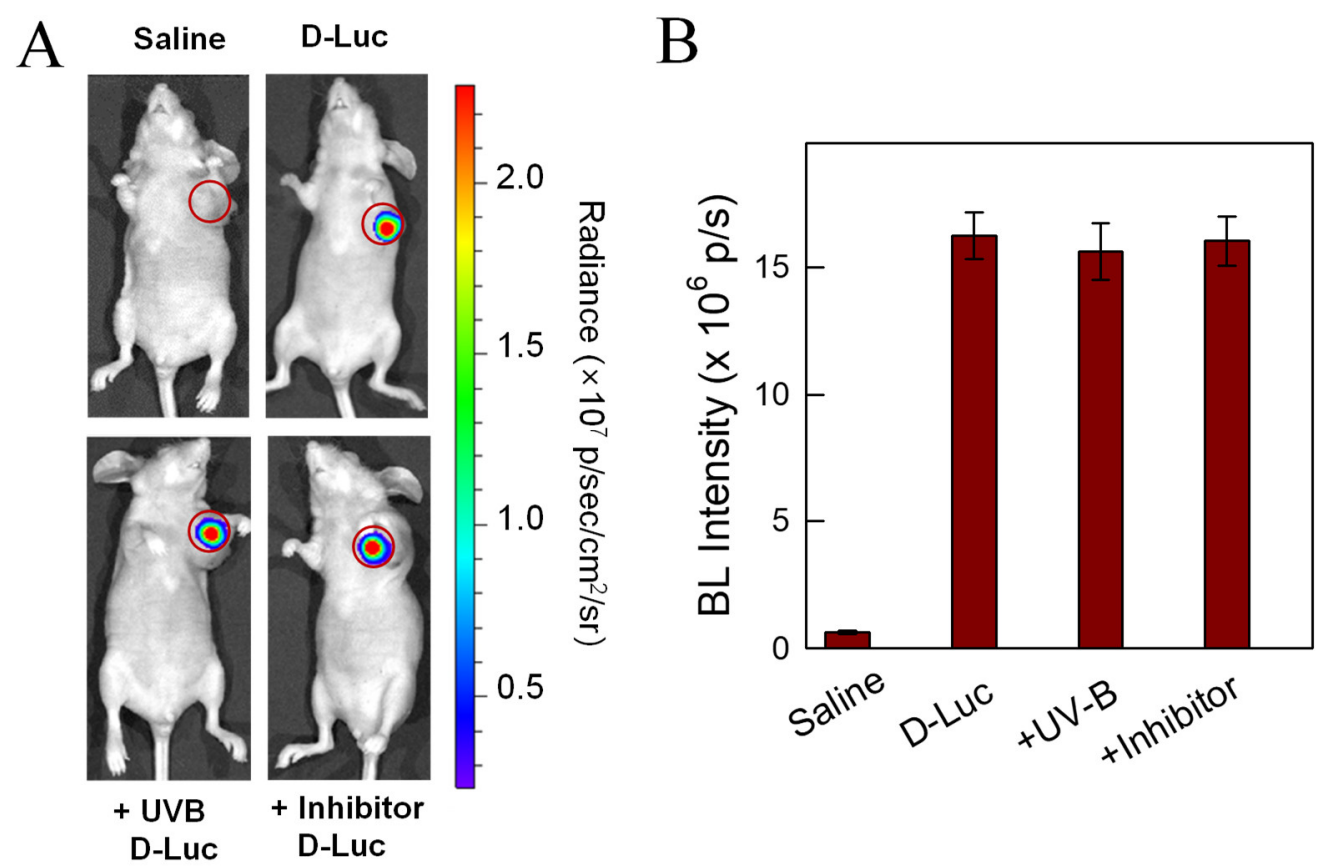

Figure S14. (A) Bioluminescence imaging of fLuc-transfected 4T1 tumor-bearing nude mice after different treatments: injection of saline or $1 \mathrm{mM}$ D-Luc; UV-B irradiation $30 \mathrm{~min}$ followed by injection of $1 \mathrm{mM}$ D-Luc; injection of $0.8 \mu \mathrm{g} / \mathrm{mL}$ indomethacin followed by $1 \mathrm{mM}$ D-Luc. (B) Quantification of the total photon flux from tumor regions of the mice in part (A). Red circles indicate the tumors. Error bars indicate s.d. $(\mathrm{n}=3)$.

Table S1. Summary of detection techniques and limits of detection of HAase or Hyal-1 detction.

\begin{tabular}{lccc}
\hline \multicolumn{1}{c}{ References } & Detection Methods & Analyte & Limit of Detection \\
\hline D-Luc@CHA in this work & bioluminescence & Hyal-1 & $0.07 \mathrm{ng} / \mathrm{mL}$ \\
Anal. Chem. 2019, $91,6887-6893$ & fluorescence & Hyal-1 & $1 \times 10^{-5} \mu \mathrm{g} / \mathrm{mL}$ \\
Chem. Commun. 2014, 50, 15696-15698 & fluorescence & HAase & $1.5 \mu \mathrm{g} / \mathrm{mL}$ \\
Anal. Chem. 2015, $87,5816-5823$ & upconversion luminescence & HAase & $0.6 \mathrm{ng} / \mathrm{mL}$ \\
\hline
\end{tabular}

